\title{
Resonances in Stark Effect and Perturbation Theory
}

\author{
Sandro Graffi ${ }^{\star} \star$ and Vincenzo Grecchi ${ }^{2} \star \star$ \\ 1 Istıtuto Matematico, Università di Modena, I-41100 Modena, Italy \\ 2 Istituto Matematico and Istituto di Fisica, Università di Modena, I-41100 Modena. Italy
}

\begin{abstract}
It is proved that the action of a weak electric field shifts the eigenvalues of the Hydrogen atom into resonances of the Stark effect, uniquely determined by the perturbation series through the Borel method.

This is obtained by combining the Balslev-Combes technique of analytic dilatations with Simon's results on anharmonic oscillators.
\end{abstract}

\section{Introduction}

The Stark effect on a Hydrogen-like atom is described by the Hamiltonian operator:

$$
H(F)=-\Delta-Z / r+2 F x_{3}
$$

acting on $L^{2}\left(R^{3}\right)$. Here $2 F>0$ is the uniform electric field directed along the $x_{3}$ axis, $Z$ the atomic number, and $r=\left(x_{1}^{2}+x_{2}^{2}+x_{3}^{2}\right)^{1 / 2}$.

As is well known, the Schrödinger operator (1.1) is a non-positive singular problem in perturbation theory of linear operators, and the spectrum of $H(F)$ is (absolutely) continuous in $(-\infty,+\infty)$, while the spectrum of the unperturbed operator $H(0)$, the Hydrogen atom, is discrete along $(-\infty, 0)$. The spectral theory of this problem is then treated within the framework of asymptotic perturbation theory [12] based upon strong convergence of resolvents as $F \rightarrow 0$.

In addition, an alternative technique for dealing specifically with this kind of problems has been very recently developed by Avron and Herbst [1] and by Veselic̀ and Weidmann [24].

It involves considering $-\Delta+2 F x_{3}$ as the unperturbed operator and $Z / r$ as the perturbation and yields the absolute continuity of the spectrum along $(-\infty,+\infty)$ and the existence of the wave operators.

By means of the strong convergence of resolvents Riddell [17] generalizing earlier results of Titchmarsh [22], proved that the spectrum of $(1.1)$ along $(-\infty, 0)$

* Partially supported by G.N.F.M., C.N.R.

$\star \star$ Partially supported by I.N.F.N., Sezione di Bologna 
is asymptotically concentrated to any order in perturbation theory, thus allowing the definition of pseudoeigenvalues to which the formal Rayleigh-Schrödinger perturbation series are asymptotic.

Here by means of the well known separability of (1.1) in squared parabolic coordinates we prove that for $F$ complex the strong resolvent convergence can be replaced by a norm resolvent convergence, in some angular sector, of a closely related operator.

This result is analogous to the one obtained by Simon [19] for the anharmonic oscillator which is an example of positive singular perturbation, and allows to strengthen purely asymptotic concepts such as spectral concentration and pseudoeigenvalues to resonances and Borel summation [9] of perturbation series.

This analogy goes actually much further, because it is well known since the first paper of Schrödinger on perturbation theory (and even earlier [6] in the framework of the old quantum theory) that the squared parabolic coordinates reduce the Hamiltonian (1.1) to a system of two constrained anharmonic oscillators with a centrifugal potential, whose quartic terms have coefficient $F$ and $-F$ respectively.

Exactly in this way Titchmarsh [23] gave a first proof of the absence of eigenvalues for (1.1) and of first order spectral concentration [22].

Here, applying the results of Simon [19] for the two-dimensional anharmonic oscillator, we see that the difficulties arising from the coefficient $-F$ can be overcome so that the Balslev-Combes [2] technique of analytic dilatations can be applied to yield the existence of resonances. In addition we prove that the resonances are uniquely determined by the divergent perturbation series through the Borel method.

The realization of (1.1) as a Hilbert space operator for $F$ complex starting from the separated ordinary differential expressions will be dealt with in the next section.

In Section III we treat the spectral theory of this operator, in Section IV we prove the existence of resonances according to both standard notions of this concept as classified by Simon [21], and in Section V the Borel summability of the divergent perturbation series.

For convenience of exposition, we collect in a very short Appendix all results we need on the quartic anharmonic oscillator with a centrifugal term.

\section{The Stark Hamiltonian: Separation of the Variables}

This section deals with the separation of the variables in the Schrödinger operator of the Stark effect. This problem, trivial at first glance, is on the contrary a fairly subtle one, as shown by Avron and Herbst [1] through an argument to be reconsidered below.

Consider again the Stark effect Hamiltonian (1.1):

$$
H=H(F)=-\Lambda-Z / r+2 F x_{3} .
$$

It is well known $[12,17]$ that the partial differential expression $(2.1)$ can be realized as an operator on $L^{2}\left(R^{3}\right)$, essentially self-adjoint on $C_{0}^{\infty}\left(R^{3}\right)=D(H)$. Its spectrum is 
absolutely continuous along $(-\infty,+\infty)[23,1]$ and its part along $(-\infty, 0)$ is asymptotically concentrated as $F \rightarrow 0$, the pseudoeigenvalues having asymptotic expansions to all orders in $F$ which coincide with the formal perturbation series [17].

As the Hydrogen atom obtained from (2.1) for $F=0$, the Stark effect Hamiltonian is separable in parabolic [13] or squared parabolic [23] coordinates. Let us briefly review the details. The squared parabolic coordinates $\xi, \eta, \phi$, $0 \leqq \xi, \eta<+\infty, 0 \leqq \phi<2 \pi$, are defined by:

$$
\begin{array}{ll}
\xi=\left(r+x_{3}\right)^{1 / 2} & x_{1}=\xi \eta \cos (\phi) \\
\eta=\left(r-x_{3}\right)^{1 / 2} & x_{2}=\xi \eta \sin (\phi) \\
\phi=\operatorname{arctg}\left(x_{2} / x_{1}\right) & x_{3}=\frac{1}{2}\left(\xi^{2}-\eta^{2}\right)
\end{array}
$$

with $r=\left(x_{1}^{2}+x_{2}^{2}+x_{3}^{2}\right)^{1 / 2}=\frac{1}{2}\left(\xi^{2}+\eta^{2}\right)$.

Then the following decomposition holds:

$$
L^{2}\left(R^{3}\right)=\bigoplus_{0}^{\infty} L_{m}, L_{m}=L^{2}\left(R_{++}^{2}, r \xi \eta d \xi d \eta\right) \otimes e^{ \pm i m \phi},
$$

where $L^{2}\left(R_{++}^{2}, r \xi \eta d \xi d \eta\right)$ denotes the Hilbert space of all (equivalence classes of) functions $f: R_{+} \times R_{+} \rightarrow C$ square integrable on $R_{+} \times R_{+}$with respect to the measure $r \xi \eta d \xi d \eta$. Then $L_{m}$ reduces $H$ for any $m$, so that $H$ can be expressed under the form of a direct sum:

$$
H=\bigoplus_{0}^{\infty} h_{m},
$$

where $h_{m}=P_{m} H P_{m}, P_{m}$ being of course the orthogonal projection from $L^{2}\left(R^{3}\right)$ to $L_{m}$.

After separation of the angular part, the problem is reduced to the study of the essentially self-adjoint operator $H_{m}^{\prime}$ on $L^{2}\left(R_{++}^{2}, r d \xi d \eta\right)$ defined by the differential expression

$$
H_{m}^{\prime}=\frac{1}{2} r^{-1}\left(-\partial^{2} / \partial \xi^{2}-\hat{o}^{2} / \partial \eta^{2}+\left(m^{2}-1 / 4\right)\left(\xi^{-2}+\eta^{-2}\right)+F\left(\xi^{4}-\eta^{4}\right)-2 Z\right)
$$

on some domain $D\left(H_{m}^{\prime}\right)$.

To investigate the spectral properties of $H_{m}^{\prime}$, consider the operator $H_{m}^{\prime}-E, E$ a complex number, whose study on $L^{2}\left(R_{++}^{2}, r d \xi d \eta\right)$ is of course equivalent to that of the operator $H_{m}$ defined on some domain $D\left(H_{m}\right) \subset L^{2}\left(R_{++}^{2}\right)$ by the differential expression

$$
H_{m}=-\partial^{2} / \partial \xi^{2}-\hat{\partial}^{2} / \partial \eta^{2}+\left(m^{2}-1 / 4\right)\left(\xi^{-2}+\eta^{-2}\right)+F\left(\xi^{4}-\eta^{4}\right)-E\left(\xi^{2}+\eta^{2}\right)-2 Z
$$

because of the unitary equivalence:

$$
\frac{1}{2}\left(\Phi,\left(H_{m}^{\prime}-E\right)^{-1} \Phi\right)=\left(r^{1 / 2} \Phi, H_{m}^{-1} r^{1 / 2} \Phi\right), \quad r^{1 / 2} \Phi \in L^{2}\left(R_{++}^{2}\right) .
$$

Formula (2.6) shows that the problem can be further separated in terms of the natural tensor product decomposition $L^{2}\left(R_{++}^{2}\right)=L^{2}\left(R_{+}\right) \otimes L^{2}\left(R_{+}\right)$. One has:

$$
H_{m}=A_{m} \otimes I+I \otimes A_{m}^{\prime}-2 Z,
$$


where $A_{m}$ and $A_{m}^{\prime}$ are the ordinary differential operators on $L^{2}\left(R_{+}\right)$defined on some corresponding domain $D\left(A_{m}\right)$ and $D\left(A_{m}^{\prime}\right)$ by:

$$
\begin{aligned}
& A_{m}=-d^{2} / d \xi^{2}+\left(m^{2}-1 / 4\right) \xi^{-2}-E \xi^{2}+F \xi^{4} \\
& A_{m}^{\prime}=-d^{2} / d \eta^{2}+\left(m^{2}-1 / 4\right) \eta^{-2}-E \eta^{2}-F \eta^{4} .
\end{aligned}
$$

(2.8) and (2.9) represent the reduction of the Stark Hamiltonian to a system of two anharmonic oscillators with a centrifugal potential. The spectrum of the Stark operator $H=H(F)$ can of course be recovered from $H_{m}$ by taking the union over $m$ of the complements of the sets of the complex $E$ plane for which $H_{m}^{-1}$ belongs to $B\left(L^{2}\left(R_{++}^{2}\right)\right)$. Therefore $H_{m}$, which shall be referred to in the following as the reduced Stark operator, has absolutely continuous generalized spectrum (in the sense of Kato [12], Chapter VII) along $(-\infty,+\infty)$ for any $m$, since the spectrum of $H=H(F)$ is absolutely continuous along $(-\infty,+\infty)$.

The subtleties arise in the inversion of this procedure, i.e. when one tries to define the Stark operator $H(F)$ on $L^{2}\left(R^{3}\right)$ starting from the separated ordinary differential expression (2.9), along the lines of the standard treatment of the Hydrogen atom in spherical or parabolic coordinates (see e.g. Hellwig [10]).

Taking as starting point the ordinary differential expressions (2.9), it is well known (see e.g. Reed and Simon [16]) that $A_{m}^{\prime}$ is in the limit circle case both at the origin and at infinity, while $A_{m}$ is in the limit circle case at the origin and in the limit point one at infinity (actually at the origin the limit circle case takes place only for $m=0$ ). Hence the deficiency indices (for $m=0$ ) of $A_{m}^{\prime}$ and $A_{m}$ defined on $C_{0}^{\infty}\left(R_{+}\right)$are $(2,2)$ and $(1,1)$, respectively, and it is known that all self-adjoint extensions of them have discrete spectrum [5]. Hence, as remarked by Avron and Herbst [1], proceeding in this way one ends up with a Stark operator which, at least for some $Z$, has a discrete spectrum. The point here is of course that the Stark operator defined in this way does not coincide with the former one defined on $C_{0}^{\infty}\left(R^{3}\right)$, because of the additional boundary conditions at infinity needed to make a self-adjoint operator in $L^{2}\left(R_{+}\right)$out of the differential expression $A_{m}^{\prime}$.

To sidestep this difficulty, in his proof of the (absolute) continuity of $\sigma(H)$ Titchmarsh [23] showed by direct construction that for $E$ complex, $\operatorname{Im}(E)>0$, there is one and only one Green's function associated with $A_{m}^{\prime}-\lambda$ if one requires only the $L^{2}$ condition at infinity. In this way for complex $E$ the operator $A_{m}^{\prime}-\lambda$ can be unambigously defined as the inverse of the Green's function. Then he showed that as $\operatorname{Im}(E) \rightarrow 0_{+}, H_{m}^{-1}$ is singular for any real $E$, thus getting the continuity of the spectrum of the (reduced) Stark operator over $(-\infty,+\infty)$. Here $H_{m}$ is defined as in (2.8). As remarked by Dolph [4], Titchmarsh's procedure is equivalent to imposing on $A_{m}^{\prime}$ for $\operatorname{Im}(E)=0_{+}$the Sommerfeld radiation condition at infinity.

Our starting point here is slightly different from Titchmarsh's one, in that we define the operators in $L^{2}\left(R_{+}\right)$out of the differential expressions $A_{m}$ and $A_{m}^{\prime}$ for complex $F, \operatorname{Im}(F)>0$, because this is needed for construction of resonances (see Section IV). The consistency with Titchmarsh's treatment will however be checked by showing that the analytic continuations of his resolvent kernels (i.e. the Green's functions) to $F$ complex, $\operatorname{Im}(F)>0$, coincide with the present ones.

After this somewhat long review, let us turn to the construction of the reduced Stark operator for $F$ complex in a region to be specified below. 
Let $F$ and $F^{\prime}$ be complex, $|\arg (F)|<\pi,\left|\arg \left(F^{\prime}\right)\right|<\pi$. For any $m=0,1 \ldots$ consider in $L^{2}\left(R_{++}^{2}\right)$ the operator defined by the following tensor product:

$$
T_{m}\left(E, F, F^{\prime}\right)=A_{m}(E, F) \otimes I+I \otimes A_{m}\left(E, F^{\prime}\right),
$$

where, for $E<0$ :

$$
A_{m}(E, F)=K_{m}(-E, F) .
$$

$K_{m}(\alpha, \beta)$ being the differential operator in $L^{2}\left(R_{+}\right)$defined in Appendix. This means that $A_{m}$ is the realization as a Hilbert space operator of the differential expression $-d^{2} / d x^{2}-E x^{2}+F x^{4}+\left(m^{2}-1 / 4\right) x^{-2}$.

Lemma 2.1. Let $|\arg (F)|<\pi,\left|\arg \left(F^{\prime}\right)\right|<\pi$, and $m=0,1, \ldots$. Then:

a) $\bar{T}_{m}\left(E, F, F^{\prime}\right)$ is a holomorphic family of type $A$ (in the sense of Kato [12]) in $E$ for any finite complex $E$, with compact resolvents.

b) The spectrum of $T_{m}\left(E, F, F^{\prime}\right)$ consists of the eigenvalues $\lambda_{h, k}^{m}=Z_{h}^{m}(E, F)$ $+Z_{k}^{m}\left(E, F^{\prime}\right), h, k=0,1, \ldots, Z_{h}^{m}(E, F)$ being the $h$-th eigenvalue of $A_{m}(E, F)$.

Proof. a) follows from assertion a) of Theorem A.1 through a trivial tensor product argument.

b) By a result of Reed and Simon [15] under the present conditions the spectral relation $\sigma\left(T_{m}\right)=\sigma\left(A_{m}(E, F)\right)+\sigma\left(A_{m}\left(E, F^{\prime}\right)\right)$ holds, and this proves Lemma 2.1 .

Corollary 2.1. Let $F$ be complex, $0<\arg (F)<\pi$, and $m=0,1, \ldots$ Then we can define

$$
T_{m}(E, F) \equiv T_{m}\left(E, F, e^{-i \pi} F\right)
$$

as an operator family on $L^{2}\left(R_{++}^{2}\right)$ enjoying properties a) and b) above. In addition $\bar{T}_{m}(E, F)$ is a holomorphic family of type $A$ in $F$ with compact resolvents.

Proof. The second assertion follows from Theorem A.1 through the tensor product argument.

Remark. Having fixed the sign convention $-F=e^{-i \pi} F$, from now on we shall simply write $-F$ to mean $e^{-i \pi} F$, whenever necessary, without further specification. As it will be seen below this convention coincides with Titchmarsh's one.

Definition 2.1. Let $F$ be complex, $0<\arg (F)<\pi$, and let $T_{m}(E, F), m=0,1, \ldots$, be as in Corollary 2.1. Then we define the reduced Stark operator $H_{m}$ for $F$ complex as:

$$
H_{m}=T_{m}(E, F)-2 Z \text {. }
$$

By spectrum of the reduced Stark operator, $\sigma\left(H_{m}\right)$, we shall mean the complement of the set of the points of the complex $E$ plane for which $\left(T_{m}(E, F)-\lambda\right)^{-1}$ at fixed $\lambda$ $=2 Z$ belongs to $B\left(L^{2}\left(R_{++}^{2}\right)\right)$.

In other words, $H_{m}$ represents the realization as an operator in $L^{2}\left(R_{++}^{2}\right)$ of the partial differential expression (2.8) for $0<\arg (F)<\pi,-F=e^{-i \pi} F$.

Lemma 2.2. For any fixed $F$ in $0<\arg (F)<\pi$, E belongs to $\sigma\left(H_{m}\right), m=0,1, \ldots$, if and only if it satisfies at least one of the conditions:

$$
Z_{h}^{m}(E, F)+Z_{k}^{m}(E,-F)=2 Z, h, k=0,1, \ldots
$$


Proof. Obvious by Corollary 2.1 .

Let us now check that our definition of the (reduced) Stark effect Hamiltonian is the correct one.

Theorem 2.1. If $E$ does not belong to $\sigma\left(H_{m}\right)$, the integral kernel of $H_{m}^{-1}=\left(T_{m}(E, F)\right.$ $-2 Z)^{-1}, H_{m}$ as in Definition 2.1, $m=0,1, \ldots$, coincides with Titchmarsh's Green's function $G_{m}$ [23], analytically continued to complex $F, 0<\arg (F)<\pi$.

Proof. We have only to check that the integral kernels of $A_{m}(E, F)^{-1}$ and $A_{m}(E,-F)^{-1}$ coincide with Titchmarsh's Green's functions $g_{m}$ and $\gamma_{m}$ continued to complex $F$, respectively. By Theorem A.1 and Formulae (A.2), this coincidence is verified by direct inspection for $E=0$, and elsewhere by the uniqueness theorem on Green's functions of ordinary differential operators, since our boundary conditions are precisely Titchmarsh's ones. This proves Theorem 2.1.

Having thus defined the reduced Stark operator for $F$ complex, $0<\arg (F)<\pi$, the Stark operator $H(F)$ on $L^{2}\left(R^{3}\right)$ for $F$ complex is specified, through (2.5)-(2.9), by the direct sum (2.4). Since $\sigma(H)$ is the union over $m$ of $\sigma\left(H_{m}\right)$, in treating the spectral theory of $H$ we can restrict our attention to $H_{m}$, which we shall do from now on.

\section{Spectrum of the Stark Operator for a Complex Field}

The definitions and the results of the former Section allow a very simple characterization of $\sigma\left(H_{m}\right)$, the spectrum of the reduced Stark effect operator, when $F$ is complex, $0<\arg (F)<\pi$.

Theorem 3.1. For any complex $Z$, and for any complex $F$ such that $0<\arg (F)<\pi$, $\sigma\left(H_{m}\right), m=0,1, \ldots$, consists of isolated eigenvalues of finite multiplicity, accumulating only at infinity.

Proof. A direct consequence of the compactness of $\left(T_{m}(E, F)-\lambda\right)^{-1}$ and of its analyticity in $E$, because of Theorem VII.1.10 of [12].

Remark. The discreteness of the spectrum of the (reduced) Stark operator for $\operatorname{Im}(F)>0$ is in sharp contrast with its absolute continuity over $(-\infty,+\infty)$ when $\operatorname{Im}(F)=0$. Remark however that the intuitive tunneling argument yielding the continuity of the spectrum [13] no more applies if $F$ is complex.

Theorem 3.1 yields no information on the analyticity of the eigenvalues of the reduced Stark operator as a function of the electric field $F$. Since some analyticity is necessary for Howland's interpretation of resonances (Section IV), and these eigenvalues are implicitly defined as functions of $F$ by the constraint $Z_{h}^{m}(E, F)+Z_{k}^{m}(E,-F)=2 Z, h, k=0,1, \ldots$, let us prove the following proposition:

Theorem 3.2. Let $m=0,1, \ldots, 0<\arg (F)<\pi$. Then there are positive constants $C(m, h, k), m, h, k=0,1, \ldots$, such that :

a) The constraint $Z_{h}^{m}(E, F)+Z_{k}^{m}(E,-F)=2 Z$ implicitly defines a family of functions $E_{h, k}^{m}(F)$ analytic in $U_{0}(m, h, k)=\{F|0<| F|<C(m, h, k)| 0<\arg (F)<\pi\}$ $m, h, k=0,1, \ldots$.

b) The functions $E_{h, k}^{m}(F), m, h, k=0,1, \ldots$, have an analytic continuation to the domain $U(m, h, k)=\{F|0<| F|<C(m, h, k)|-\pi / 2+\varepsilon<\arg (F)<3 \pi / 2-\varepsilon \mid \varepsilon>0\}$ across the cut at $F$ real. 
c) As $F \rightarrow 0, F \in U(m, h, k)$, the function $E_{h, k}^{m}(F)$ tends to the Hydrogen atom eigenvalue of magnetic quantum number $m$ and parabolic quantum numbers $h$ and $k$.

This provides a natural ordering for the eigenvalues as far as no level crossing takes place, which is true in the sectors specified above by a) and b).

The proof of this theorem is based upon the following Lemma 3.1. Remark that from now on we drop the index $m$, since the subsequent arguments do not depend on it.

Lemma 3.1. Let $p_{h, k}(y)=\left(Z_{h}(1, y)+Z_{k}(1,-y)\right) /(2 Z), h, k=0,1, \ldots$ Then $p_{h . k}(y)$ is analytic in the region $U_{2}(h, k)=\{y|0<| y|<B(h, k)|-\pi / 2+\varepsilon<\arg (y)<3 \pi / 2-\varepsilon \mid \varepsilon>0\}$ cut along the real axis. Here $B(h, k)=\min (B(h), B(k)), B(h)$ as in Theorem A.1. Moreover for $y$ in $U_{2}(h, k)$ :

$$
\begin{aligned}
& p_{h, k}(y) \equiv p_{0}(h, k)+p_{1}(h, k) y+0\left(y^{2}\right), \quad \text { for } \quad h \neq k, \\
& p_{h, h}(y) \equiv q_{h}\left(y^{2}\right)=q_{0}(h)+q_{1}(h) y^{2}+0\left(y^{4}\right), \quad \text { for } \quad h=k .
\end{aligned}
$$

$p_{0}, p_{1}, q_{0}, q_{1}$, being real constants, with $p_{0}>0, q_{0}>0, p_{1} \neq 0, q_{1} \neq 0$.

Proof. Analyticity of $p_{h, k}(y)$ in $U_{2}(h, k)$ is an immediate consequence of Theorem A.1. Furthermore, Theorem A.1 implies a fortiori that the perturbation series is asymptotic to all orders to $Z_{h}(1, y)$, uniformly for $y$ in $U_{h}, U_{h}$ as in Theorem A.1. Then (3.1) and (3.2) simply follow by the non vanishing of the perturbation coefficients, which is true because $x^{4}$ is a positive perturbation of $p^{2}+x^{2}+$ $\left(m^{2}-1 / 4\right) / x^{2}$, a positive operator.

Proof of Theorem 3.2. By the Symanzik scaling (see Simon [19]), $Z_{h}(-E, F)$ $=w^{-1} Z_{h}\left(-E w^{-2}, F w^{-3}\right)$ for $E<0, w>0$, and elsewhere by analytic continuation. Then, with $w=(-E)^{-1 / 2}$, the constraint equation $Z_{h}(-E, F)+Z_{k}(-E,-F)=2 Z$ becomes:

$$
p\left(F w^{3}\right)-w=0,
$$

where the indices $h$ and $k$ have been omitted for simplicity.

Beginning by $h \neq k$, let us first show that if a solution $w=w(F)$ of (3.3) exists as an analytic function of $F$ in a region $U$ with $C \leqq B$, then:

$$
w(F)=p_{0}+0(F), F \in U .
$$

For, $w(F)$ must then satisfy $w=p\left(F w^{3}\right)$, which by (3.1) yields:

$$
w=p_{0}+0\left(F w^{3}\right)=p_{0}+p_{0}^{3} 0(F)
$$

when $F w^{3} \in U_{2}$, and hence $F \in U$ for $C$ small enough because $\arg \left(w^{3}\right)=0(F)$.

By means of (3.3) the explicitation problem for $w=w(F)$ can be solved in the following way : first by inverting $p\left(F w^{3}\right)$ one finds $F$ as a function of $w$, and then by inverting this last function one finds $w$ as a function of $F$. Lemma 3.1 ensures, as we shall see, that the invertibility conditions [14] are fulfilled.

Now the function $F_{1}(w)=w^{-3} p^{-1}(w)$ solves $(3.3)$ if $p^{-1}(w)$ exists. $p^{-1}(w)$ in turn exists and is analytic in some sector $p\left(U_{2}\right)$, for $B$ small enough, since $d p / d y=p_{1}$ $+0(y) \neq 0$ by continuity in some sector $U_{2}$ if $B$ is chosen suitably small. Now $F_{1}(w)$ can be inverted to yield a function $F_{1}^{-1}(F)=w(F)$ analytic in $U$ because $d F_{1} / d w$ 
$=w^{-3} d p^{-1} / d w-3 w^{-4} p^{-1}(w)=w^{-3} d p^{-1} / d w-3 w^{-1} F_{1}(w)=\left(p_{0}\right)^{-3}\left(p_{1}\right)^{-1}+0(F)$ by (3.5), and this quantity does not vanish, by continuity, when $F$ is in $U$ for $C$ small enough. Finally, again by $(3.5), E(F)=-(w(F))^{-2}$ exists and is analytic in $U$. For $h=k$ one has only to replace $p(y)$ by $q\left(y^{2}\right)$ and to apply (3.2) instead of (3.1). This proves a) and b). To see c), notice that this assertion is equivalent to the continuity of the functions $E_{h, h}^{m}(F)$ as $F \rightarrow 0, F \in U$. Again this is true by the continuity of $p(y)$ as $y \rightarrow 0$ in $U_{2}(h, k)$, and this concludes the proof of Theorem 3.2.

As an immediate consequence we have:

Corollary 3.1. Let $F$ be fixed, $F \in U_{0}=\{F|0<| F|<C| 0<\arg (F)<\pi\}$. Then $\sigma\left(H_{m}\right)$ contains as eigenvalues of finite multiplicity the analytic functions $E_{h, k}^{m}(F)$ for all $h, k$ such that $C(h, k) \geqq C$.

\section{Analytic Continuation of Eigenvalues to Resonances}

In this section we shall see how the former results on spectral properties of the (reduced) Stark operator for complex $F$ allow the application of the BalslevCombes [2] technique of analytic dilatations. In this way the existence of resonances will be proved, according to both standard notions of this concept for self-adjoint operators, i.e. the Livsic-Grossmann and the Friedrichs-Howland ones (see Simon [21] for an illuminating discussion on this point).

Let us begin by recalling some notions of the Balslev-Combes technique, strictly necessary in what follows.

Let $U_{1}(\theta), \theta \in R$, be the group of unitary dilatations in $L^{2}\left(R^{3}\right)$ defined by:

$\left(U_{1}(\theta) f\right)(\boldsymbol{r})=e^{3 \theta / 2} f\left(e^{\theta} \boldsymbol{r}\right), f \in L^{2}\left(R^{3}\right)$.

The unitary group $U_{1}(\theta)$ is generated by the skew self-adjoint operator $A_{1}=\frac{1}{2}(\boldsymbol{r} \cdot \boldsymbol{\nabla}+\boldsymbol{\nabla} \cdot \boldsymbol{r}), U_{1}(\theta)=e^{\theta A_{1}}$.

A vector $\phi_{1} \in L^{2}\left(R^{3}\right)$ is dilatation analytic if it is an analytic vector in the sense of Nelson for the generator $A_{1}$ of the unitary dilatations $U_{1}(\theta)$, i.e. if $\sum_{0}^{\infty}\left\|A_{1}^{n} \phi_{1}\right\| \theta^{n} / n !<+\infty$ for all finite $\theta \in C$. The set $\left\{\phi_{1}\right\}$ of all dilatation analytic vectors is dense in $L^{2}\left(R^{3}\right)$.

To apply these notions to the present case of the reduced Stark operator $H_{m}$, first remark that the unitary group $U_{1}(\theta)$ induces in a natural way a unitary group $U(\theta)$ in $L^{2}\left(R_{++}^{2}\right)$, specified by:

$$
(U(\theta) f)(u, v)=e^{1 / 2 \theta} f\left(e^{1 / 2 \theta} u, e^{1 / 2 \theta} v\right), f \in L^{2}\left(R_{++}^{2}\right) .
$$

$U(\theta)$ is of course generated by the skew self-adjoint operator $A=\frac{1}{2}(\boldsymbol{x} \cdot \boldsymbol{\nabla}+\boldsymbol{\nabla} \cdot \boldsymbol{x})$, $x \in R_{++}^{2}, \boldsymbol{\nabla}$ the two-dimensional gradient operator. Then, as before, $\phi$ is a dilatation analytic vector if $\sum_{0}^{\infty}\left\|A^{n} \phi\right\| \theta^{n} / n !<\infty$, and the set $\{\phi\}$ is dense.

For $\theta \in R, 0<\arg (F)<\pi, m=0,1, \ldots$, let us now define on $L^{2}\left(R_{++}^{2}\right)$ the operator

$$
T_{m}(E, F, \theta)=U(\theta) T_{m}(E, F) U(\theta)^{-1}
$$

and, accordingly:

$$
H_{m}(\theta)=U(\theta) H_{m} U(\theta)^{-1}=U(\theta)\left(T_{m}(E, F)-2 Z\right) U(\theta)^{-1} .
$$


One has trivially:

$$
\begin{aligned}
& T_{m}(E, F, \theta)=e^{-\theta} T_{m}\left(E e^{2 \theta}, F e^{3 \theta}\right), \\
& H_{m}(\theta)=e^{-\theta}\left(T_{m}\left(E e^{2 \theta}, F e^{3 \theta}\right)-2 Z e^{\theta}\right) .
\end{aligned}
$$

We now have to extend these definitions to complex $\theta$.

An immediate consequence of the results of Section III is the following:

Lemma 4.1. The operator $T_{m}(E, F, \theta)$ has an analytic continuation to the strip $-\arg (F) / 3<\operatorname{Im}(\theta)<\pi / 3-\arg (F) / 3$. By this we mean that for these values of $\theta T_{m}(E, F, \theta)$ is defined on $D_{m} \otimes D_{m}$ and has a compact resolvent which is analytic in $\theta$.

Proof. It is enough to remark that if we define $F^{\prime}=F e^{3 \theta}, E^{\prime}=E e^{2 \theta}$, one has $0<\arg \left(F^{\prime}\right)<\pi$ when $\theta$ belongs to the above strip. Then an application of Lemma 2.1 and Corollary 2.1 proves the present Lemma.

Corollary 4.1. Let $\theta$ belong to the strip $0<\operatorname{Im}(\theta)<\pi / 3$, and let $F$ be real. Then the eigenvalues of $H_{m}(\theta)$, which by Lemma 4.1 has compact resolvent, do not depend on 0 .

Proof. By (4.6) the eigenvalues of $H_{m}(\theta)$ are given by those values of $E$ which satisfy the constraint

$$
Z_{h}\left(E e^{2 \theta}, F e^{3 \theta}\right)+Z_{k}\left(E e^{2 \theta},-F e^{3 \theta}\right)=2 Z e^{\theta}
$$

and thus do not depend on $\theta$ by the scaling property.

This proves the Corollary.

Remark. These eigenvalues are thus resonances of the (reduced) Stark effect Hamiltonian according to the Livsic-Grossmann notion as defined by Simon [21].

Let us now proceed to verify that these eigenvalues are resonances also according to the Friedrichs-Howland notion (see always Simon [21]).

Theorem 4.1. Let $\Psi \in L^{2}\left(R_{++}^{2}\right)$ be a dilatation analytic vector. Then the function $f_{\Psi}(E)=\left(\Psi,\left(H_{m}(E, F)\right)^{-1} \Psi\right), F$ real, originally defined as an analytic function of $E$ in the upper half-plane $\operatorname{Im}(E)>0$, has a meromorphic continuation to the lower halfplane $\operatorname{Im}(E)<0$ across the cut $-\infty<E<+\infty$.

The poles of $f_{\Psi}(E)$ for $\operatorname{Im}(E)<0$ are the eigenvalues of $H_{m}(\theta), 0<\operatorname{Im}(\theta)<\pi / 3$.

Proof. For $F$ complex, $\operatorname{Im}(F)>0$, consider the function:

$$
f_{\Psi}(E)=\left(\Psi,\left(H_{m}(E, F)\right)^{-1} \Psi\right)=\left(\Psi,\left(T_{m}(E, F)-2 Z\right)^{-1} \Psi\right)
$$

which by the results of Section III exists and is analytic in $E$ as long as $2 Z$ is different from all eigenvalues of $T_{m}(E, F)$. For $\theta \in R$ we have, with $\Psi(\theta)=U(\theta) \Psi$ :

$$
f_{\Psi}(E)=\left(\Psi(\theta),\left(T_{m}(E, F, \theta)-2 Z\right)^{-1} \Psi(\theta)\right)
$$

and this equality holds by analytic continuation also for all $\theta$ in the strip $-\arg (F) / 3<\operatorname{Im}(\theta)<\pi / 3-\arg (F) / 3$, because of Lemma 4.1 and of the fact that $\Psi(\theta)$ has an analytic continuation to the strip, $\Psi$ being a dilatation analytic vector. If we now restrict $\theta$ to the strip $0<\operatorname{Im}(\theta)<\pi / 3-\varepsilon, \varepsilon>0$, for any fixed value of $\theta$ we 
can perform the analytic continuation of (4.9), and hence of (4.8), to $F$ real from above, for all values of $E$ except those satisfying (4.7). To conclude the proof, it remains to check analyticity for $\operatorname{Im}(E)>0$. Since analyticity of $f_{\Psi}(E)$ for $\operatorname{Im}(E)>0$ and absence of eigenvalues for $\operatorname{Im}(E)=0$ are precisely Titchmarsh's results, the above values of $E$ satisfying (4.7) must lie in the lower half-plane $\operatorname{Im}(E)<0$, and this ends the proof of Theorem 4.1.

Remarks. a) The analyticity of $f_{\Psi}(E)$ for $\operatorname{Im}(E)>0$ can be proved directly, by showing that $\operatorname{Im} \bar{E}(F)>0$ is impossible for $F$ real. Here $\bar{E}(F)$ is any solution of $Z_{h}(E, F)+Z_{k}(E,-F)=2 Z$. For, if this is possible, we would have: $\operatorname{Im} Z_{1}=$ $-\operatorname{Im} \bar{E}\left(\%, x^{2} \chi\right) /(\chi, \chi)<0$, where $Z_{1}$ is an eigenvalue $Z_{h}(E, F)$ with $F>0$. On the other hand for $\arg (F)>0$ one has:

$$
\operatorname{Im} Z_{2}=-\operatorname{Im} \bar{E}\left(\omega, x^{2}(\omega)\right) /(\omega, \omega)+\operatorname{Im}\left(F e^{-i \pi}\right)\left(\omega, x^{4} \omega\right) /(\omega, \omega)<0,
$$

where $Z_{2}$ is an arbitrary eigenvalue $Z_{k}(E,-F) \cdot \chi$ and $\omega$ are the eigenvectors corresponding to $Z_{1}$ and $Z_{2}$, respectively. Hence $\lim \left(\operatorname{Im} Z_{2}\right)$ as $\arg (F) \rightarrow 0_{+}$, which under the present conditions exists at least as a measure by a theorem of Herglotz [19], cannot be positive. Therefore $\operatorname{Im}\left(Z_{h}+Z_{k}\right)<0$ if $F$ is real, and the constraint $Z_{h}+Z_{k}=2 Z$ cannot be satisfied.

b) The eigenvalues $E_{h, k}(F)$ of the (reduced) Stark operator which are analytic in $U_{0}(h, k)$ can be continued to $F$ real, as we know from Theorem 3.2. At $F$ real by Theorems 4.1 and 4.2 they are resonances according to both notions. Hence the functions $E_{h . k}(F)$ realize Howland's mechanism for the onset of resonances [11]: i.e. they are analytic continuations to second sheet poles of the resolvent of a selfadjoint operator of functions which represent actual eigenvalues of the operator family when $F$ is complex.

\section{Borel Summability of Perturbation Series}

We turn now to the determination of those resonances given by the analytic continuation of the eigenvalues $E_{h, k}(F)$ through the time-independent (i.e. Rayleigh-Schrödinger) perturbation theory, although it gives rise, as we shall see, to divergent expansions. This will be achieved by proving the Borel summability of the Rayleigh-Schrödinger series to the functions $E_{h, k}(F)$ for $F$ complex, $\operatorname{Im}(F)>0$, where they are eigenvalues of the (reduced) Stark operator. Since the resonances are the analytic continuation of the eigenvalues to $F$ real, they turn out to be uniquely determined by Rayleigh-Schrödinger perturbation theory.

Let us begin by stating a further consequence of the implicit function argument of Theorem 3.2 under the form of a Lemma.

Lemma 5.1. Let $\left\{E_{h, k}(F)\right\}_{h, k=0}^{\infty}$ be the family of the eigenvalues of the (reduced) Stark operator analytic in $U(h, k)$. Then any $E_{h, k}(F)$ has a formal Taylor expansion $\sum_{0}^{\infty} S_{i} F^{i}$ near $F=0$. Here $S_{i} \neq 0$ for all $i$ if $E_{h, k}(F)$ is implicitly defined by $Z_{h}+Z_{k}=2 Z$ with $h \neq k$, and $S_{2 i+1}=0$ if it is defined for $h=k$.

Proof. The existence of the Taylor expansion near $F=0$ follows again from the analogous statement valid for $Z_{h}(1, F)$ through the implicit function argument of 
Section III. The assertion on the coefficients is a direct consequence of the simple remark:

$$
p(y)=Z_{h}(1, y)+Z_{k}(1,-y) \sim \sum_{0}^{\infty}\left(a_{n}^{h}+(-1)^{n} a_{n}^{k}\right) y^{n} \equiv \sum_{0}^{\infty} p_{n} y^{n}
$$

with $a_{n}^{h} \neq a_{n}^{k}$ if $h \neq k$ as it is easily checked. This proves Lemma 5.1.

Remarks. a) The Taylor expansions of Lemma 5.1 are formal. The non-analyticity of $E_{h, k}(F)$ at $F=0$ is not enough to prove divergence. Actually the divergence, not faster than $n$ !, follows here from the Borel summability, as we shall see below.

b) The power series $\sum_{0}^{\infty} S_{i} F^{l}$ coincides with the Rayleigh-Schrödinger perturbation expansion, as proved by Titchmarsh [22] up to first order, and by Riddell [17] to any order.

Let us now quote the standard criterion yielding the Borel summability [9].

Theorem (Watson). Let $F(x)$ be analytic in a sector $D(C, \varepsilon)$ on the Riemann surface of $\log (x)$ given by $D(C, \varepsilon)=\{x|0<| x|<C||\arg (x)| \leqq \pi / 2+\varepsilon\}$ for some $C>0, \varepsilon>0$. Let $F(x)$ admit the power series $\sum_{0}^{\infty} a_{n} x^{n}$ as an asymptotic expansion as $x \rightarrow 0$ in $D(C, c)$. Then the series $\sum_{0}^{\infty} a_{n} x^{n}$ is Borel summable to $F(x)$ in the sector $D_{1}(C, \varepsilon)=$ $=\{x|0<| x|<C||\arg (x)| \leqq \varepsilon\}$, if there are positive constants $A, B$ independent of $x$ such that for all $x$ in $D(C, \varepsilon)$ :

$$
\left|R_{N}(x)\right| \leqq A B^{N} N ! x^{N}
$$

Here $R_{N}(x)=F(x)-\sum_{0}^{N-1} a_{n} x^{n}$ is the $N$-th order remainder of the expansion $\sum_{0}^{\infty} a_{n} x^{n}$. Remarks.

a) (5.1) implies a fortiori $\left|a_{n}\right| \leqq A B^{n} n$ !

b) Let us recall that the Borel summability of $\sum_{0}^{\infty} a_{n} x^{n}$ to $F(x)$ has the following meaning: the function $F_{B}(x)=\sum_{0}^{\infty} a_{n} x^{n} / n$ !, the Borel transform, which by $(5.2)$ is analytic for $|x|<1 / B$, has an analytic continuation into the whole sector $|\arg (x)| \leqq \varepsilon$, such that the integral

$$
\int_{0}^{\infty} e^{-a} F_{B}(x a) d a
$$

converges uniformly and absolutely and coincides with $F(x)$ in $D_{1}(C, \varepsilon)$.

To apply this criterion to our case we need a simple generalization, stated as:

Lemma 5.2. Let $F(x)$ and $\sum_{0}^{\infty} a_{h} x^{k}$ fulfill the conditions of Watson's theorem. Then the same is true for $F^{n}(x)=(F(x))^{n}$ and $\left(\sum_{0}^{\infty} a_{k} x^{k}\right)^{n} \equiv \sum_{0}^{\infty} a_{k}^{(n)} x^{k}, n=2,3 \ldots$, i.e. there is $L>0$ 
independent of $x, L / A=\sup _{J} \sum_{0}^{J} k !(J-k) ! / J !$, such that :

$$
\left|R_{N}^{(n)}(x)\right|=\left|F^{n}(x)-\sum_{0}^{N-1} a_{k}^{(n)} x^{k}\right| \leqq A L^{n} B^{N} N !|x|^{N}, \quad N=1,2, \ldots
$$

Proof. The assertion is trivial for $n=2$, and a simple inductive argument shows its validity for any $n$.

Remarks. a) $\sum_{0}^{\infty} a_{k}^{(n)} x^{k}=\left(\sum_{0}^{\infty} a_{k} x^{k}\right)^{n}$ is then Borel summable to $F^{n}(x)$ in $D_{1}(C, \varepsilon)$.

b) If $F(x) \neq 0$ in $D(C, \varepsilon)$ this result can be proved also for negative $n$ [18].

c) If for $n \leqq 3 N$ we set $B^{\prime}=B L^{3}$ in (5.4) we have:

$$
\left|R_{N}^{(n)}(x)\right| \leqq A\left(B^{\prime}\right)^{N} N !|x|^{N}, \quad N=1,2, \ldots
$$

Theorem 5.1. Let $F(x)$ and $\sum_{0}^{x} a_{n} x^{n}$ be as above, and $a_{0}>0$. Then if the equation $F\left(x w^{3}\right)=w$ implicitly defines a function $w(x)$ analytic in a sector $D\left(C^{\prime}, \varepsilon\right), 0<C^{\prime} \leqq C$, with an asymptotic expansion near $x=0$ in $D\left(C^{\prime}, \varepsilon\right)$, the expansion is Borel summable to $w(x)$ in $D_{1}\left(C^{\prime}, \varepsilon\right)$.

Proof. If there is a solution $w(x)$ analytic in $D\left(C^{\prime}, \varepsilon\right)$ with an asymptotic expansion $\sum_{0}^{\infty} b_{n} x^{n}$ such that

$$
\left|R_{N}^{(1)}(x)\right| \leqq A_{1} B_{1}^{N} N !|x|^{N}, \quad N=1,2 \ldots M-1,
$$

where $R_{N}^{(1)}(x)=w(x)-\sum_{0}^{N-1} b_{n} x^{n}$, uniformly for $x$ in $D\left(C^{\prime}, \varepsilon\right)$, then (5.5) holds for all $N$. For, by induction (5.5) holds for all $N$ if it holds for $N=1$, and this is true by the very existence of the implicit function $w$ as an analytic function of $x$ in $D\left(C^{\prime}, \varepsilon\right)$, which is in turn true because $\partial\left(F\left(x w^{3}\right)-w\right) / \hat{o} w=-1+0(x) \neq 0$ in $D\left(C^{\prime}, \varepsilon\right)$ for $C^{\prime}$ small enough.

Let us now proceed to the verification of (5.5). Let $\sum_{0}^{\infty} b_{n, k} x^{n}$ be the asymptotic expansion of $w^{k}(x)$. Then if $k \leqq 3(M-1)$ by Remark c) after Lemma 5.2 we have:

$$
\left|R_{N}^{(k)}(x)\right|=\left|w^{k}(x)-\sum_{0}^{N-1} b_{n, k} x^{n}\right| \leqq A^{\prime}\left(B^{\prime}\right)^{N} N !|x|^{N}, \quad N=2, \ldots, M
$$

for some positive constants $A^{\prime}$ and $B^{\prime}$ independent of $x$ in $D\left(C^{\prime}, \varepsilon\right)$. Substituting into the equation $F\left(x w^{3}\right)-w=0$ we get the following relations:

$$
\sum_{0}^{M-1} b_{n} x^{n}+R_{M}^{(1)}(x)=a_{0}+\sum_{1}^{M-1} a_{n} x^{n}\left(\sum_{0}^{M-n-1} b_{k, 3 n} x^{k}+R_{M-n}^{(3 n)}(x)\right)+R_{M}\left(x w^{3}\right)
$$

which are satisfied for:

$$
\begin{aligned}
& b_{0}=a_{0}, b_{n}=\sum_{0}^{N-1} b_{j, 3(n-j)} a_{n-j}, \quad n=1,2, \ldots, M-1 \\
& R_{M}^{(1)}(x)=\sum_{0}^{M-1} a_{n} x^{n} R_{M-n}^{(3 n)}(x)+R_{M}\left(w^{3} x\right) .
\end{aligned}
$$


Therefore there are constants $A^{\prime \prime}$ and $B^{\prime \prime}$ independent of $x$ such that $\left|R_{M}^{(1)}(x)\right|$ $\leqq A^{\prime \prime}\left(B^{\prime \prime}\right)^{M} M !|x|^{M}$ for all $x$ in $D\left(C^{\prime}, \varepsilon\right)$, because $\left|R_{M}\left(x w^{3}\right)\right| \leqq a A B^{M} M !|x|^{M}$ in $D(C, \varepsilon)$, with a suitable $a>0$ independent of $w$, since $\arg \left(w^{3}\right)$ is small for $C^{\prime}$ small enough. Hence by Watson's theorem $\sum_{0}^{\infty} b_{n} x^{n}$ is Borel summable to $w(x)$ in $D_{1}\left(C^{\prime}, \varepsilon\right)$, and this proves Theorem 5.1 .

Corollary 5.1. The (Rayleigh-Schrödinger) perturbation expansion of any function $E_{h, k}^{m}(F)$, analytic in $U(m, h, k)$, which represents an eigenvalue of the (reduced) Stark operator for $\operatorname{Im}(F)>0$ and a resonance for $F$ real, is Borel summable to $E_{h, k}^{m}(F)$ in the region $\{F|0<| F|<C(m, h, k)| \eta<\arg (F)<\pi-\eta\}$.

Proof. By Theorem A.1c), Theorem 3.2 and Lemma 5.1, with $\varepsilon=\frac{1}{2} \pi-\eta$ we can apply Theorem 5.1 to all functions $\bar{F}(x) \equiv p(y), x=-i y$, and all implicit functions $\bar{w}(x) \equiv w(F), x=-i F$. The result then follows from Lemma 5.2, Remark b), because $w(F) \rightarrow p_{0}>0$ in $U$, and $E(F)=-\left((w(F))^{-2}\right)$.

Remark. The one-to-one relationship between any function $E_{h, k}^{m}(F)$ and its perturbation series provided by the Borel summability implies the divergence of the series itself. For, if it would converge, the function $E_{h . k}^{m}(F)$ would be real for $F$ real within the convergence circle since all coefficients are real, and this is impossible.

\section{Appendix}

Consider the differential expression $p^{2}+\alpha x^{2}+\beta x^{4}+\left(m^{2}-1 / 4\right) x^{-2}, m=0,1, \ldots$ We shall denote by $K_{m}(\alpha, \beta)$ its realization as an operator on $L^{2}\left(R_{+}\right)$. This differential expression can be obtained in a straightforward way from the twodimensional anharmonic oscillator

$$
-\Delta+\alpha\left(x^{2}+y^{2}\right)+\beta\left(x^{2}+y^{2}\right)^{2}
$$

if one changes to polar coordinates and restricts to angular momentum $m$, $m=0,1, \ldots$. Hence if one defines out of $(\mathrm{A} .1)$ an operator in $L^{2}\left(R^{2}\right)$ on the domain $\mathscr{S}\left(R^{2}\right)$, a direct application of the results of Simon ([19], Section III) yields for $K_{m}(\alpha, \beta)$, defined on a corresponding domain $D_{m} \subset L^{2}\left(R_{+}\right)$:

Theorem A.1. $K_{m}(\alpha, \beta)$ is an operator family in $L^{2}\left(R_{+}\right)$enjoying, for any $m=0,1, \ldots$, the following properties:

a) For any $\beta$ in the cut plane $|\arg (\beta)|<\pi$, and any complex $\alpha, K_{m}(\alpha, \beta)$ is a closable operator with $\bar{K}_{m}(\alpha, \beta)^{*}=\bar{K}_{m}(\bar{\alpha}, \bar{\beta})$, and compact resolvent. For any fixed $\beta$ in the cut plane, the resolvent of $K_{m}(\alpha, \beta)$ is analytic in $\alpha$, for any complex $\alpha$.

b) Let $m$ and $i$ be given non-negative integers, and $\eta<3 \pi / 2$. Then there is a $B=B(m, i)>0$ such that any eigenvalue $\lambda_{i}^{m}(1, \beta)$ of $K_{m}(1, \beta)$ is analytic in $\{\beta \mid \beta$ on a three-sheeted Riemann surface $|0<| \beta|<B,| \arg (\beta) \mid<\eta\}$.

c) The Rayleigh-Schrödinger perturbation expansion of any eigenvalue $\hat{i}_{i}^{m}(1, \beta)$ is divergent and Borel summable to $\hat{\lambda}_{i}^{m}(1, \beta)$ in the region $\{\beta|0<| \beta|<B||\arg (\beta)|$ $<\pi-\varepsilon, \varepsilon>0\}$. 
Remark. Assertion c) is actually proved in [8] and [20]. Finally, the resolvent kernel of $K_{m}(0, \beta),|\arg (\beta)|<\pi$, is given, as it is easily checked, by the following Green's function:

$$
G_{m}(x, y)= \begin{cases}f_{m}(x) g_{m}(y) & 0 \leqq x \leqq y<+\infty \\ f_{m}(y) g_{m}(x) & 0 \leqq y \leqq x<+\infty\end{cases}
$$

where $f_{m}(x)=\sqrt{x} I_{m / 3}\left(\sqrt{\beta} x^{3} / 3\right), g_{m}(x)=\sqrt{x} K_{m / 3}\left(\sqrt{\beta} x^{3} / 3\right) . I_{\mu}$ and $K_{v}$ are the Bessel functions of the third kind $[3,7]$.

Acknowledgements. It is a pleasure to thank Professors S. Albeverio, J. M. Combes, A. Grossmann, and R. Höegh-Krohn for their interest in this work and useful discussions.

\section{References}

1. Avron,J.E., Herbst,I.W.: Commun. math. Phys. 52, 239 (1977)

2. Balslev, E., Combes, J.M.: Commun. math. Phys. 22, 280 (1971)

3. Buchholz,H.: The confluent hypergeometric function. Berlin, Heidelberg, New York: Springer 1967

4. Dolph,C.L.: Bull. A.M.S. 67, 1 (1961)

5. Dunford, N., Schwartz,J.T. : Linear operators, Vol. II. New York: Interscience 1958

6. Epstein,P.S.: Ann. Physik (Leipzig) (4), 50, 489 (1916)

7. Gradshteyn,I.S., Ryzhik,I.M.: Tables of integrals, series, and products. New York, London: Academic Press 1965

8. Graffi, S.. Grecchi, V., Simon, B. : Phys. Lett. 32B, 631 (1970)

9. Hardy,G.H.: Divergent series. Oxford: Oxford University Press 1947

10. Hellwig,G.: Differentialoperatoren der mathematischen Physı. Berlin, Göttingen, Heidelberg, New York: Springer 1964

11. Howland,J.: Pacific J. Math 55. 157 (1974)

12. Kato, T.: Perturbation theory for linear operators. Berlin, Heidelberg, New York: Springer 1966

13. Landau, L.D., Lifshitz,E.M.: Quantum mechanics. Oxford: Pergamon Press 1958

14. Markushevich, A.: Teoria de las funciones analiticas. Editorial Mir (1970)

15. Reed, M., Simon, B.: Bull. A.M.S. 78. 730 (1970)

16. Reed,M., Simon, B.: Methods of modern mathematical physics, Vol. II. New York, London: Academic Press 1975

17. Riddell, R.C.: Pacific J. Math. 23, 377 (1967)

18. Simon, B.: Phys. Rev. Letters 28, 1145 (1972)

19. Simon, B.: Ann. Phys. 58, 76 (1970)

20. Simon, B.: Advan. Math. 7, 240 (1971)

21. Simon, B.: Ann. Math. 97, 247 (1973)

22. Titchmarsh,E.C.: J. Anal. Math. 4, 187 (1955)

23. Titchmarsh,E.C.: Proc. London Math. Soc. (3), 5, 1 (1955)

24. Veselic̀, K., Weidmann,J.: Math. Z. 156, 93 (1977)

Communicated by J. Ginibre

Received February 9, 1978 